\title{
Chronic rhinosinusitis and omalizumab: eosinophils not lgE predict treatment response in real-life*
}

Jagdeep Sahota ${ }^{1,3}$, Therese Bidder ${ }^{1,2}$, Rebecca Livingston', Douglas S. Robinson', Valerie J. Lund ${ }^{2,3}$, Matthew P. Su ${ }^{3}$, Harsha H. Kariyawasam², ${ }^{1,3}$

'Thoracic Medicine, University College London Hospital NHS Foundation Trust, London, United Kingdom

2 Rhinology Section, Royal National Throat Nose and Ear Hospital London, London, United Kingdom

${ }^{3}$ University College London, London, United Kingdom
Rhinology Online, Vol 1: 147 - 153, 2018

http://doi.org/10.4193/RHINOL/18.077

${ }^{*}$ Received for publication:

September 18, 2018

Accepted: October 8, 2018

Published: October 15, 2018

\begin{abstract}
Background: Chronic rhinosinusitis with and without nasal polyps (CRSwNP and CRSsNP respectively) can be difficult to treat and biologics offer potential as a future therapeutic intervention. CRS and severe asthma often co-exist. Omalizumab is currently licenced for the treatment of severe allergic asthma.
\end{abstract}

Objective: The aim was to determine which blood biomarkers and treatment variables were associated with clinical response for sinonasal symptoms in patients with allergic CRSsNP and CRSwNP who were treated with omalizumab for severe allergic asthma. Methods: Sinonasal outcome test (SNOT)-22 scores were recorded in a cohort of patients with allergic CRSsNP ( $n=10$ ) or CRSwNP $(n=15)$ treated with omalizumab for severe asthma according to UK guidelines. Using a linear modelling approach, the improvement in the SNOT-22 score as the treatment variable against several disease and treatment predictor variables was modelled.

Results: SNOT-22 significantly decreased with omalizumab treatment. Increased blood eosinophil count and omalizumab dosing every two weeks (as opposed to every 4 weeks) were associated with greater reductions in SNOT-22 after 16 weeks of omalizumab treatment.

Conclusion: Our study confirms that omalizumab is an effective treatment for CRS, and suggests that improvements are greater in those with eosinophilic disease. Further studies are required to determine long term efficacy and whether this treatment could reduce the need for surgery.

Key words: omalizumab, nasal polyps, asthma, biomarkers, lgE, eosinophils, outcomes

\section{Introduction}

Chronic rhinosinusitis (CRS) is an umbrella term for a group of heterogeneous diseases featuring inflammation of the nose and sinuses. It can be further sub-classified into those with or without nasal polyps (CRSwNP and CRSsNP respectively) ${ }^{(1)}$. Sinus disease is found in nearly $90 \%$ of mild to moderate asthmatics and in up to $100 \%$ of severe steroid-dependent asthmatics as evidenced on sinonasal imaging ${ }^{(2)}$, emphasising the considerable burden this syndrome can place on healthcare resources and reduction in quality of life. CRS associated with asthma pheno-endotypes often demonstrate, in the broadest sense, a common immune signature defined by Th2 T cell predominance
(3). The hallmarks of such $\mathrm{T} 2$ inflammation includes interleukin (IL)-5 driven eosinophil recruitment and survival, IL-4 induction of immunoglobulin E ( $\mathrm{lgE}$ ) synthesis and IL-13 augmenting this Th2 driven inflammation. Serum IgE is a reflection of excess unbound tissue $\lg E$ in washout blood and secreted $\lg E$ from circulating lgE+ plasma cells (albeit a smaller proportion)(4). Studies have shown that blood eosinophilia correlates with high tissue eosinophilia in CRS ${ }^{(5)}$ and patients with both high blood $\mathrm{lgE}$ and eosinophils have a more aggressive disease phenotype resistant to treatment ${ }^{(6,7)}$. Furthermore, individuals who have CRSwNP have been shown to have elevated tissue IgE and eosinophils ${ }^{(8)}$. This is consistent with the one airway hypothesis 
as asthma often coexists; severe asthmatics tend to have high serum eosinophil counts and IgE together ${ }^{(9)}$, reflecting the same immunopathological process occurring throughout the airway. High levels of these blood biomarkers indicate high T2 inflammatory tissue states, and are used in severe asthma treatment protocols to select patients for more precise target specific treatment using lgE inhibition and eosinophil depletion strategies respectively.

Omalizumab (Xolair ${ }^{\circledR}$ Novartis) is an anti-lgE monoclonal lgG1 antibody licenced for therapeutic use in severe allergic asthma. It binds to free serum IgE. The site of binding at the $\mathrm{C} \varepsilon 3$ domain prevents the interaction of IgE with Fc\&RI via steric hindrance, preventing mast cell and basophil activation and degranulation ${ }^{(10)}$. There is subsequent downregulation of FccRl due to the decrease in free IgE levels ${ }^{(11)}$. Omalizumab also prevents $\mathrm{IgE}$ binding to the low affinity IgE receptor CD23 on B cells and antigen-presenting cells ${ }^{(10)}$. A consequence of blocking this $\lg E$ mediated cell signalling is a decrease in IL-5 production ${ }^{(12)}$ and cellular release. Hence recruitment of bone marrow derived eosinophils into blood and tissue sites is decreased as is eosinophil survival resulting in eosinophil depletion ${ }^{(13-15)}$. Thus with time the $T 2$ inflammatory axis is downregulated ${ }^{(16)}$.

In the UK, currently only those with a raised blood IgE within a set range matched for body weight and a clearly identifiable perennial aeroallergen with severe allergic asthma can be treated with omalizumab. We previously reported our real-life experience with a group of $n=13$ severe allergic asthmatic patients with co-existent CRSwNP successfully treated with omalizumab (17). Briefly our findings were that both CRSwNP and asthma improved in parallel, with the improvement in CRSwNP comparable to functional endoscopic sinus surgery with polypectomy at their 16-week follow-up. All the CRSwNP patients demonstrated raised total blood IgE and a significant proportion also had a raised eosinophil count (despite at least 4 courses of oral steroids to stabilise their asthma within the 12 months prior to omalizumab). To evaluate what predictor variables can potentially identify upper airway treatment response with omalizumab, we have incorporated the data of another further 2 CRSwNP patients giving data on a total 15 patients with CRSwNP and data on 10 patients with just allergic CRSsNP. Increased combined patient numbers allowed us to set a more robust linear model system. Linear models describe a continuous response variable as a function of one or more predictor variables. We evaluated whether the dose of omalizumab, frequency of omalizumab administration, starting severity of CRS (as defined by the baseline sinonasal outcome test (SNOT)-22 score), actual diagnosis of CRSwNP or the biomarkers blood IgE total or blood eosinophil count was likely to be associated with the omalizumab treatment response. Here we report our findings.

\section{Materials and Methods}

Ethics approval and consent to participate

This study did not require ethics approval or consent as the data was collected prospectively as part of the clinical assessment of asthma and is presented in an anonymised manner.

\section{Availability of data and material}

The datasets used and/or analysed during the current study are available from the corresponding author on reasonable request.

A cohort of patients with severe allergic asthma as defined in the ERS/ATS published criteria underwent detailed upper airway assessment as part of an asthma investigation protocol ${ }^{(18)}$. All were sensitised to common perennial aeroallergens. Based on direct nasal endoscopic examination and clinical history, patients were defined as either having allergic CRSsNP or CRSwNP. The UK prescribing criteria for omalizumab in severe refractory allergic asthma (selecting those individuals most likely to respond to an anti-lgE blocking approach) is as follows: a) confirm sensitisation to a perennial aeroallergen such as house dust mite (HDM) or cat; b) total blood lgE equal to or within 30 to $1500 \mathrm{lU} /$ $\mathrm{ml}$ within a specific weight group to match. The dosing chart dictates the dose of omalizumab and whether it is to be administered subcutaneously every 2 or 4 weeks. A mandatory omalizumab efficacy-of-treatment assessment in asthma is required at 16 weeks, and this visit was used to evaluate any sinonasal treatment response using the SNOT-22 score in a prospective manner. The SNOT-22 is the recommended outcome tool in adult CRS in the European Position Paper on Rhinosinusitis and Nasal Polyps $2012^{(1)}$. It provides information on both symptoms and the burden of disease on general health as used in several other publications assessing outcomes in CRS. The SNOT-22 was therefore used as the measure of disease severity and the improvement in SNOT-22 as the continuous response variable as a function of one or more predictor variables stated here. All patients were treated with nasal isotonic saline volume douching, intranasal steroids and if indicated a prolonged immunomodulatory antibiotic before commencing omalizumab. In addition, oral steroids were administered to stabilise disease in individuals with exacerbation prone or severe asthma. Thus, the baseline SNOT-22 scores documented just prior to the first dose of omalizumab reflect fully treated, optimised asthma and sinonasal disease. Subsequent changes in the SNOT-22 score correspond with improvements following omalizumab administration in CRS. This is, therefore, reflective of real-life clinical data, and describes the additional effects omalizumab has on medically maximised CRS treatment.

\section{Statistical analysis}

Data is expressed as the median (range) or standard error (SE) as stated. All paired within-subject data was analysed using the 
Table 1. Summary of patient characteristics and baseline biomarkers.

\begin{tabular}{lcc|}
$\begin{array}{l}\text { Demography }+ \\
\text { Biomarkers }\end{array}$ & CRSsNP & CRSwNP \\
\hline Age (years) & $45.5(20-63)$ & $51(25-66)$ \\
\hline Sex & $\mathrm{F} \mathrm{n=6} \mathrm{M} \mathrm{n=4}$ & $\mathrm{F} \mathrm{n=8} \mathrm{M} \mathrm{n=7}$ \\
\hline $\begin{array}{l}\text { Perennial allergen } \\
\text { sensitisation }\end{array}$ & $100 \%$ & $100 \%$ \\
\hline $\begin{array}{l}\text { Aspirin sensitisation } \\
\text { Severe asthma }\end{array}$ & $0 \%$ & $75 \%$ \\
\hline Eosinophils cells/ $\mu \mathrm{L}$ & $210(40-840)$ & $100 \%$ \\
\hline Blood lgE total IU/mL & $195.5(90-1463)$ & $350(60-3060)$ \\
\hline SNOT-22 & $18.50(4-44)$ & $52.50(10-84)$ \\
\hline
\end{tabular}

Values are stated as the median (range) where appropriate. $\mathrm{F}=\mathrm{female}$ $M=$ male.
Table 2. Summary of predictor variables included in the linear model.

\begin{tabular}{|rc|}
\hline \multicolumn{1}{|c|}{ Linear Variable Data } & Value \\
\hline SNOT-22 score at baseline & $40.0 \pm 4.59$ \\
Eosinophil value $<150$ cells/ $\mu \mathrm{L}$ & $6(24 \%)$ \\
$>150$ cells/ $\mu \mathrm{L}$ & $19(76 \%)$ \\
\hline Blood lgE total value $<200 \mathrm{IU} / \mathrm{mL}$ & $9(36 \%)$ \\
$>200 \mathrm{IU} / \mathrm{ml}$ & $16(64 \%)$ \\
\hline Four weekly treatment regimens & $17(68 \%)$ \\
Two weekly treatment regimens & $8(32 \%)$ \\
\hline Omalizumab $=150 \mathrm{mg}$ dosage & $6(24 \%)$ \\
$>150 \mathrm{mg}$ dosage & $19(76 \%)$ \\
\hline
\end{tabular}

given as either median \pm SE or absolute patient numbers with percentages of the overall group size
Wilcoxon signed rank test. Significance was accepted as $\mathrm{p}<0.05$. A generalised linear model was created with the SNOT-22 score at 16 weeks as the response variable. Since SNOT-22 scores at 16 weeks were not normally distributed, the natural logarithm of these scores was taken; this was then found to be normally distributed.

The decision to include predictor variables in the model was determined via expert judgement of clinical significance and calculation of the Akaike information criterion (AIC) values for each model, with the most parsimonious model (as determined by the AIC value) being used for the final analysis. The predictor variables included in the final model were: SNOT-22 score at baseline, blood IgE total value, blood eosinophil value, patient diagnosis of CRSWNP (on the assumption more severe patients will respond better to omalizumab), and dosage regimen in terms of both injection frequency and drug amount.

As the $\lg E$ and eosinophil values were found to be non-normally distributed, these parameters were split into two groups. For $\lg \mathrm{E}$ values, $<200$ (baseline) and $\geq 200 \mathrm{IU} / \mathrm{mL}$ were used, whilst for eosinophil values $<150$ (baseline) and $\geq 150$ cells/ $\mu \mathrm{L}$ were used. These cut-off points were taken from previously published reports ${ }^{(19,20)}$. Patients were grouped as allergic CRSsNP when there was no definite visible polyps on endoscopic examination with CRS who gave a clear history of allergen induced exacerbation of sinonasal symptoms.

Participants were diagnosed with either allergic CRSsNP (baseline) or CRSwNP polyps. Participants could either be treated once every two or every four weeks (baseline). Dosage groups were defined as either receiving 150mg (baseline) or $>150 \mathrm{mg}$ per dose. The final model had an R-squared score of 0.586 , adjusted R-squared score of 0.4553 and an F-statistic of 4.482 on 6 and 19 DF; this corresponds to a p-value of 0.005 , suggesting that the model fits the data significantly better than a random model. All statistical analysis was done using either GraphPad
Prism version 4 (GraphPad Software Inc., San Diego, CA, USA) or the R software package [R Core team, 'R: A Language and Environment for Statistical Computing, (2018), URL = https://www.Rproject.org]. All tests for normality used Shapiro-Wilk Normality tests with a significance level of $p<0.05$. Data collected from 25 patients was used for all statistical analyses.

\section{Results}

The patient demography is summarised in Table 1. In total, complete data sets were available for 25 patients with CRS and severe allergic asthma ( $n=10$ with allergic CRSsNP and $n=15$ with (RSwNP). Of this group $n=9$ patients were on prednisolone at treatment entry till the 16-week assessment visit. Table 2 summaries the predictor variables included in the linear model analysis.

For the allergic CRSsNP group the 16-week SNOT-22 decreased from 18.50 (range 4-44) to $12.00 \pm(3-32)(p=0.058)$ whilst in the CRSWNP cohort the SNOT-22 decreased from 52.50 (range $10-84)$ to $31.50 \pm(1-95)(p=0.007)$. The SNOT-22 remained significantly decreased in the total combined group from baseline after 4 months of omalizumab therapy $(p<0.001)$ at $23.0 \pm 4.37$ (Figure $1 \mathrm{~A}$ ), with a median decrease of 16 points \pm 3.89 . Only 2 patients recorded an increased SNOT-22 score. The median serum eosinophil count significantly decreased from pre-omalizumab levels at 16 weeks $(p=0.007)$. Post-treatment eosinophils counts were only available in $n=12$ patients of whom $n=4$ were on a fixed dose of prednisolone pre-omalizumab until the 16 week assessment visit.

Table 3 summarises the output of linear model data with standard variables being calculated as shown. Of the blood biomarkers, only the predictor variable of blood eosinophil count of $\geq 150$ cells $/ \mu \mathrm{L}$, was found to have a significant effect on the SNOT-22 response variable $(p=0.03)$ and corresponded to an estimated decrease of $63.9 \%$ in the SNOT-22 score as compared 
Table 3. Summary of output of linear model, with starred variables being calculated as significant.

\begin{tabular}{lccccc}
\multicolumn{1}{c}{ Predictor variable } & Estimate & $\begin{array}{c}\text { \% change in de- } \\
\text { pendent variable }\end{array}$ & Std. Error & t value & p (>|t|) \\
\hline SNOT-22 score at baseline & 0.008869 & $0.8908 \%$ & 0.011568 & 0.767 & 0.45 \\
\hline IgE group $\geq 200 \mathrm{IU} / \mathrm{mL}$ & 1.398656 & $304.9753 \%$ & 0.766524 & 1.825 & 0.08 \\
\hline *Eosinophil group $\geq 150$ cells/ $\mu \mathrm{L}$ & -1.017978 & $-63.8675 \%$ & 0.428687 & -2.375 & 0.03 \\
\hline Diagnosis of CRSwNP & 0.659054 & $93.2962 \%$ & 0.500108 & 1.318 & 0.20 \\
\hline *Two weekly dosage regimens & -1.478457 & $-77.2011 \%$ & 0.463712 & -3.188 & 0.005 \\
\hline 150mg per dose & -0.914187 & $-59.9158 \%$ & 0.760283 & -1.202 & 0.24 \\
\hline
\end{tabular}

to having a count of $<150$ cells/ $\mu \mathrm{L}$ (Figure 1B). Baseline total blood IgE did not predict any future improvement in SNOT-22 score after omalizumab treatment. Furthermore, a two weekly omalizumab dosing regimen clearly demonstrated clinical efficacy ( $p=0.005$ ). Increasing the frequency of dosing from once every four weeks to once every two weeks led to an estimated 77.2\% decrease in SNOT-22 score from baseline (Figure 1B).

\section{Discussion}

Our real-life observations confirm that omalizumab improves sinonasal outcomes in patients with high-T2 inflammatory CRS pheno-endotypes. Despite patient selection in severe allergic asthma and CRS for omalizumab being purely based on the blood total IgE level, with sensitisation to at least one perennial aeroallergen being present, our analysis suggests that the biomarker of blood eosinophil count, rather than blood IgE total level, is a better predictor of treatment response to omalizumab in CRS.

Given the rapidly emerging market of biologics for $\mathrm{T} 2 \mathrm{high}$ asthma syndromes and CRS, being able to predict response to biologic therapy is essential. The aim of such 'precision medicine' is to target patients with a drug that will work to improve treatment outcomes quickly but also constrain costs by preventing wastage of agents and delivery resources. Biomarker guided therapy may help identify such CRS specific, treatment responsive subgroups. Our finding of blood eosinophil counts as opposed to total blood lgE predicting treatment response to omalizumab in CRS is consistent with other asthma omalizumab treatment studies. These also show an improved treatment response in relation to eosinophil counts, rather than just IgE levels (21, 22). Such disease biomarker association of treatment response between asthma and co-existing CRS provides further support for the 'united airway' concept and highlight the presence of overlapping inflammatory mechanisms between the upper and lower airway.

We are aware of the limitations of our observational data and further work with greater numbers of CRS patients is needed before any definite assumptions can be made. It is clear that future modelling efforts will need to incorporate more data and clearer groupings with composite biomarkers such as serum IL-5 and eosinophil count. In the interests of consistency between studies and to further explore the characteristics of these CRSasthma pheno-endotypes, it would be useful for future doubleblind studies to incorporate more detailed sinonasal endoscopic grading, pre- and post-treatment; radiological documentation using the Lund-Mackay score will aid this. Our findings again open the important debate for the urgent need of further robust clinical studies which utilise broader biomarker patient groups, when undertaking studies of current and emerging immunomodulatory biologics in airway disease. The concept that omalizumab neutralises unbound perennial aeroallergen specific $\operatorname{lgE}$ and inhibits any allergen-lgE activated immune cascade is probably far too simplistic an explanation for its mechanism of action. Omalizumab works equally well in asthmatics sensitised to either pollens or perennial allergens ${ }^{(23)}$. Omalizumab efficacy in non-allergic asthma, urticaria and eosinophilic oesophagitis further emphasises its ability to modulate complex immune cascades in so called 'non-allergic states' (24-27).

Omalizumab treatment leads to both blood and tissue eosinophil levels to rapidly reduce as previously stated. Our findings that patients with high blood eosinophils have a better clinical response to omalizumab are therefore not totally unexpected. Eosinophils or at least IL-5 clearly have a role in the pathogenesis of CRS as shown by IL-5 blockade studies with mepolizumab $(28,29)$. Another point worth considering is the effect of low dose prednisolone in patients with CRS and asthma and their clinical response to omalizumab. Prednisolone can modulate immune responsive factors, such as preventing eosinophilic exacerbations and this may have worked to modulate the benefits of omalizumab. Unfortunately, given our low patient numbers, with recurrent prednisolone use before omalizumab, it is difficult to delineate the exact nature of such prednisolone and omalizumab interactions on the clinical response. We stress this is real-life data, and some patients will always need treatment combinations.

As far as we are aware, specific IgE levels to an aeroallergen does not predict the efficacy of omalizumab treatment. Given that blood IgE does not reflect tissue IgE concentrations ${ }^{(4,8)}$, and 
A)

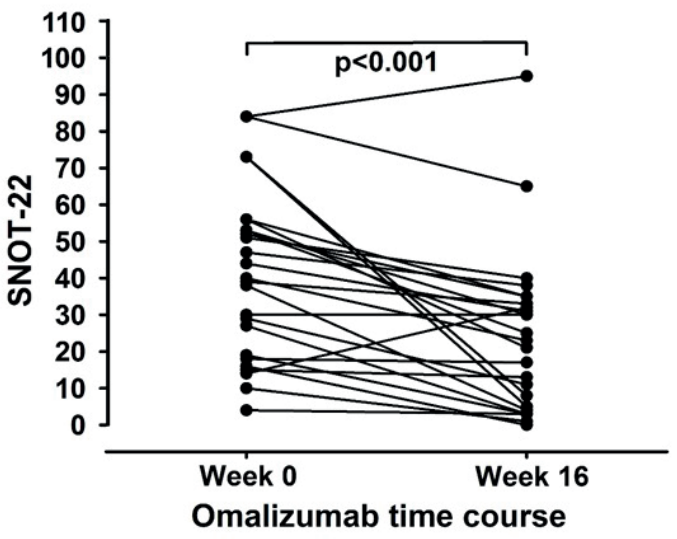

B)

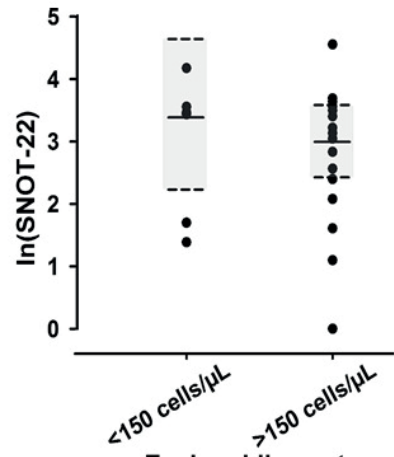

Eosinophil count

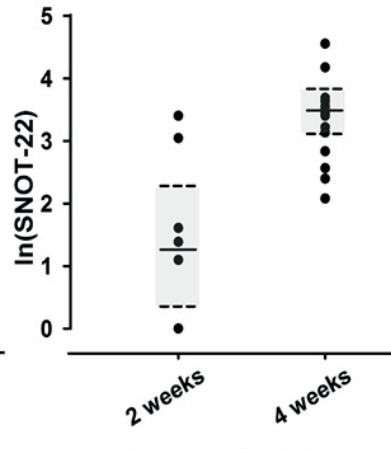

Dosing schedule
Figure 1. A) Summary of the time course of the treatment response in CRS to omalizumab in terms of SNOT-22 before the first dose of Omalizumab and at the 16th week of treatment. B) Visualisation of the relationship between the reduction of the response variable SNOT-22 and the significant predictor variables baseline blood eosinophils and omalizumab frequency. The bars represent median values for each group whilst the grey shaded areas represent confidence intervals. Individual data points represent SNOT-22 scores of individual patients in each predictor variable group at the 16 th week of omalizumab treatment.

nasal polyp disease is not necessarily driven by specific aeroallergens such as HDM, it follows that blood lgE as a biomarker for T2-CRS may not be clinically relevant. CRSwNP with aspirin sensitivity is highly eosinophilic, and such high serum eosinophilia will indicate higher $\mathrm{T} 2$ disease where both high tissue lgE and eosinophils are present. Our allergic CRSsNP group had lower levels of serum eosinophils and responded less well to omalizumab.

What is more difficult to explain is the finding that more frequent omalizumab dosing led to a better CRS treatment outcome. Twice monthly dosing has been shown to be efficacious in difficult urticaria ${ }^{(30)}$. At present we can only speculate and may have something to do with the pharmacokinetics of IgE interaction with its receptor. The current literature confirms that it is when omalizumab lowers unbound blood free lgE to less than $20 \mathrm{IU} / \mathrm{ml}$ that clinical benefit is seen in allergic asthmatic patients ${ }^{\left({ }^{11}\right)}$. Omalizumab reduces serum-free $\lg \mathrm{E}$ in a dose-related manner ${ }^{(32)}$. With this in mind, dosing protocols have been developed using information gleaned from complex pharmacodynamic modelling approaches, in order for the optimal level of $\lg E$ inhibition to be achieved and ensure all free blood $\lg E$ is bound and 'inactivated' by omalizumab. However, as the serum elimination half-life of omalizumab averaged 26 days ${ }^{(33)}$ and the half-life of IgE binding to FceRI is 14-21 days, half the $\lg E$ is free after this time to bind new receptors and potentially re-initiate immune inflammatory cascades ${ }^{(34,35)}$. Thus, injecting omalizumab at 2 weekly intervals may capture this newly released $\mathrm{IgE}$ and prevent re-FcERI binding. Another explanation could be through the allosteric actions of omalizumab on IgE where higher levels of omalizumab can lead to a phenomenon termed accelerated dissociation ${ }^{(36)}$. Here, at least in-vitro omalizumab can bind to receptor-bound $\lg \mathrm{E}$ and cause dissociation of the $\lg \mathrm{E}$ from FceRI (although at concentrations much higher than those used therapeutically). It has similarly been shown to occur on cells, but at present, it is not known to what extent, if any, this effect contributes to the therapeutic efficacy of omalizumab. If this process also occurs in-vivo in theory, lgE binding to FcERI is destabilized which would result in rapid dissociation of IgE from FceRI. This may lead to the loss of IgE- FceRI induced cellular activation and hence less inflammation downstream. Further work and discussion is clearly needed.

\section{Conclusion}

In conclusion we report that omalizumab can improve CRS outcomes where there is a high $\mathrm{T} 2$ inflammatory disease signal of raised blood eosinophils present. It is not necessarily disease severity that predicts treatment response as having CRSWNP (i.e. more severe symptoms) alone did not seem to be associated with clinical improvement. Our understanding of disease-relevant biomarkers and dosing regimens that best predict treatment response to biologics in CRS is clearly at an early stage. Further studies are needed, and it is essential that researchers study pheno-endotypes with broader multiple overlapping biomarker groups when testing the efficacy of biologics. This will ensure that large cohorts of potentially treatment responsive patients are not excluded from such therapeutic approaches, as currently is the case. Studies must also look at long term treatment efficacy and any reduced need for surgery. Important questions to answer will be whether biologics will be an alternative treatment to surgery or used in conjunction with surgery to prevent disease recurrence. In addition, the health economics of treating associated multisystem disease such as CRS and asthma with one treatment must be carefully considered. Only then can precision medicine be delivered in an effective and cost-constrained manner ${ }^{(37)}$. 


\section{Authorship contribution}

JS, TB, RL and HHK undertook data collection. MPS was institutional statistician and with HK undertook data-analysis. DSR, VJL and HHK undertook results interpretation and supervision of study. All authors contributed to the writing of the manuscript.

\section{Conflict of interest}

JS, TB, RL, VJL, MPS have no conflict of interest. DSR and HHK have undertaken paid consultancy work for Novartis.

\section{References}

1. Fokkens WJ, Lund VJ, Mullol J et al: European Position Paper on Rhinosinusitis and Nasal Polyps 2012. Rhinol Suppl 2012:3298.

2. Bresciani M, Paradis L, Des RA et al: Rhinosinusitis in severe asthma. J Allergy Clin Immunol 2001, 107:73-80.

3. Akdis CA, Bachert C, Cingi $C$ et al: Endotypes and phenotypes of chronic rhinosinusitis: a PRACTALL document of the European Academy of Allergy and Clinica Immunology and the American Academy of Allergy, Asthma \& Immunology. J Allergy Clin Immunol 2013, 131:1479-1490.

4. Eckl-Dorna J, Pree I, Reisinger J et al.: The majority of allergen-specific IgE in the blood of allergic patients does not originate from blood-derived B cells or plasma cells. Clin Exp Allergy 2012, 42:1347-1355.

5. Sreeparvathi A, Kalyanikuttyamma LK, Kumar M, Sreekumar N, Veerasigamani N: Significance of Blood Eosinophil Count in Patients with Chronic Rhinosinusitis with Nasal Polyposis. J Clin Diagn Res 2017, 11: Mc08-mc11.

6. Baroody FM, Suh SH, Naclerio RM: Total IgE serum levels correlate with sinus mucosal thickness on computerized tomography scans. J Allergy Clin Immunol 1997, 100:563568.

7. Aslan F, Altun E, Paksoy S, Turan G: Could Eosinophilia predict clinical severity in nasal polyps? Multidiscip Respir Med 2017, 12:21.

8. Bachert C, Gevaert P, Holtappels G, Johansson SG, Van Cauwenberge P: Total and specific lgE in nasal polyps is related to local eosinophilic inflammation. J Allergy Clin Immunol 2001, 107:607-614.

9. Haughney J, Morice A, Blyth KG, Lee AJ, Coutts A, McKnight $E$ et al: A retrospective cohort study in severe asthma describing commonly measured biomarkers: Eosinophil count and IgE levels. Respir Med 2018, 134:117-123.

10. Wright JD, Chu HM, Huang $\mathrm{CH}$, Ma C, Chang TW, Lim C: Structural and Physical Basis for Anti-IgE Therapy. Sci Rep 2015, 5:11581.

11. Gomez G, Jogie-Brahim S, Shima M Schwartz LB: Omalizumab reverses the phenotypic and functional effects of IgEenhanced Fc epsilonRI on human skin mast cells. J Immunol 2007, 179:1353-1361.

12. Takaku Y, Soma T, Nishihara F et al: Omalizumab attenuates airway inflammation and interleukin-5 production by mononuclear cells in patients with severe allergic asthma. Int Arch Allergy Immunol 2013, 161
Suppl 2:107-117.

13. Skiepko R, Zietkowski Z, Lukaszyk M et al: Changes in blood eosinophilia during omalizumab therapy as a predictor of asthma exacerbation. Postepy Dermatol Alergol 2014, 31:305-309.

14. Noga O, Hanf G, Brachmann I et al: Effect of omalizumab treatment on peripheral eosinophil and T-lymphocyte function in patients with allergic asthma. J Allergy Clin Immunol 2006, 117:1493-1499.

15. van Rensen EL, Evertse CE, van Schadewijk WA et al: Eosinophils in bronchial mucosa of asthmatics after allergen challenge: effect of anti-lgE treatment. Allergy 2009, 64:7280

16. Djukanovic R, Wilson SJ, Kraft M et al: Effects of treatment with anti-immunoglobulin $E$ antibody omalizumab on airway inflammation in allergic asthma. Am J Respir Crit Care Med 2004, 170:583-593.

17. Bidder T, Sahota J, Rennie C, Lund VJ, Robinson DS, Kariyawasam $\mathrm{HH}$ Omalizumab treats chronic rhinosinusitis with nasal polyps and asthma together-a real life study. Rhinology 2018, 56:42-45.

18. Chung KF, Wenzel SE, Brozek JL et al: International ERS/ATS guidelines on definition, evaluation and treatment of severe asthma. Eur Respir J 2014, 43:343-373.

19. Carosso A, Bugiani M, Migliore E, Anto JM DeMarco R: Reference values of total serum $\lg \mathrm{E}$ and their significance in the diagnosis of allergy in young European adults. Int Arch Allergy Immunol 2007, 142:230-238

20. Nair P, O'Byrne PM: Measuring Eosinophils to Make Treatment Decisions in Asthma. Chest 2016, 150:485-487.

21. Casale TB, Chipps BE, Rosen K, Trzaskoma B Haselkorn T, Omachi TA: Response to omalizumab using patient enrichment criteria from trials of novel biologics in asthma. Allergy 2018, 73:490-497.

22. Humbert M, Taille C, Mala L, Le Gros V, Just J, Molimard M: Omalizumab effectiveness in patients with severe allergic asthma according to blood eosinophil count: the STELLAIR study. Eur Respir J 2018, 51

23. Domingo $C$, Pomares $X$, Navarro $A$ et al. Omalizumab is Equally Effective in Persistent Allergic Oral CorticosteroidDependent Asthma Caused by Either Seasonal or Perennial Allergens: A Pilot Study. Int J Mol Sci 2017, 18

24. Gevaert P, Calus L, Van Zele $T$ et al: Omalizumab is effective in allergic and nonallergic patients with nasal polyps and asthma. J Allergy Clin Immunol 2013, 131:110-
116

25. Maurer M, Rosen K, Hsieh HJ, Saini S Grattan C, Gimenez-Arnau A: Omalizumab for the treatment of chronic idiopathic or spontaneous urticaria. N Engl J Med 2013, 368:924-935.

26. Simon D, Cianferoni A, Spergel JM et al: Eosinophilic esophagitis is characterized by a non-lgE-mediated food hypersensitivity. Allergy 2016, 71:611-620.

27. Loizou D, Enav B, Komlodi-Pasztor E et al: A pilot study of omalizumab in eosinophilic esophagitis. PLoS One 2015, 10: e0113483.

28. Gevaert P, Van Bruaene N, Cattaert T, van Steen K, Van Zele T, Acke F: Mepolizumab, a humanized anti-IL-5 mAb, as a treatment option for severe nasal polyposis. J Allergy Clin Immunol 2011, 128:989-995.

29. Bachert C, Sousa AR, Lund VJ et al: Reduced need for surgery in severe nasal polyposis with mepolizumab: Randomized trial. J Allergy Clin Immunol 2017, 140:1024-1031. e1014.

30. Turk M, Kocaturk E, Cure K, Yilmaz I: Twoweek intervals during omalizumab treatment may provide better symptom contro in selected patients with chronic urticaria. Allergy Clin Immunol Pract 2018.

31. Hochhaus G, Brookman L, Fox $\mathrm{H}$ et al Pharmacodynamics of omalizumab: implications for optimised dosing strategies and clinical efficacy in the treatment of allergic asthma. Curr Med Res Opin 2003, 19:491498.

32. Casale TB: Anti-immunoglobulin E (omalizumab) therapy in seasonal allergic rhinitis. Am J Respir Crit Care Med 2001, 164: S1821.

33. Lowe PJ, Tannenbaum S, Gautier A, Jimenez $P$ : Relationship between omalizumab pharmacokinetics, IgE pharmacodynamics and symptoms in patients with severe persistent allergic (lgE-mediated) asthma. Br J Clin Pharmacol 2009, 68:61-76.

34. Geha RS, Helm B, Gould H: Inhibition of the Prausnitz-Kustner reaction by an immunoglobulin epsilon-chain fragment synthesized in E. coli. Nature 1985, 315:577-578.

35. Sutton BJ, Davies AM: Structure and dynamics of IgE-receptor interactions: FcepsilonR and CD23/FcepsilonRII. Immunol Rev 2015, 268:222-235.

36. Davies AM, Allan EG, Keeble AH et al: Allosteric mechanism of action of the therapeutic anti-IgE antibody omalizumab. J Biol Chem 2017, 292:9975-9987.

37. Lund VJ, Hopkins C, Akdis C et al. EUFOREA Rhinology Research Forum 2017: report of 
the brainstorming sessions on endotypedriven treatment, patient empowerment and digital future in airways care. Rhinology Online, Vol 1: 11-19, 2018.
Dr Harsha Kariyawasam, PhD FRCP

Rhinology Section

Adult Specialist Allergy and Clinical Immunology

Royal National Throat Nose Ear Hospital and UCLH

330 Grays Inn Road

London WC1X 8DA

United Kingdom

Tel: +44 2034565242

Fax +44 2034565183

E-mail:

harsha.kariyawasam@nhs.net 